\title{
Photosynthesis of grape leaves with 'OSC' trellis and cordon based on data model fitting
}

\author{
F.C. ZHANG ${ }^{*, \dagger}$, H.X. ZHONG ${ }^{*}$, , X.M. ZHOU*, S.A. HAN* M. WANG ${ }^{*}$, J.Z. HAO ${ }^{* *}$ (D), X.Y. WU ${ }^{*,+}$, \\ and M.Q. PAN ${ }^{*},+$ \\ Institute of Horticultural Crops, Xinjiang Academy of Agricultural Science/Scientific Observing and Experimental \\ Station of Fruits in Xinjiang, Ministry of Agriculture of China, 830091 Urumqi, Xinjiang, China* \\ Institute of Plant Protection, Xinjiang Academy of Agricultural Sciences, 830091 Urumqi, Xinjiang, China**
}

\begin{abstract}
The photosynthetic parameters of 'Flame Seedless' and 'Red Globe' grape leaves in oblique single cordon (OSC) vine along the ditch and traditional single cordon (TSC) vine were determined using the CIRAS-2 photosynthetic system. The photosynthesis of leaves was studied using the Gaussian multi-peak model, Yezipiao's model, and the extended Freundlich's model fitting methods. The results showed that the correlation coefficients of the three data models fitting the field data were between 0.89 and 0.97 . The three models can be used to analyze photosynthesis of grape leaves. In this paper, the physical significance of indicators obtained by extended Freundlich's model fitting was verified. The grape leaves of OSC showed higher daily accumulation of photosynthesis, maximum photosynthetic rate, saturated light intensity, light adaptation range, and light-use efficiency than that of TSC. Grape leaves under OSC can better adapt to the environment.
\end{abstract}

Keywords: extended Freundlich's model; photosynthetic parameters; slope; viticulture; Vitis vinifera.

\section{Introduction}

Models are used in research on plant photosynthesis for light-response analysis (Prado and De Moraes 1997, Ye et al. 2013a,b), the response to $\mathrm{CO}_{2}$ (Bassman and Zwier 1991, Harley et al. 1992, Ethier and Livingston 2004), the response to temperature (Bernacchi et al. 2003), chlorophyll (Chl) fluorescence (Bordenave et al. 2019, Norton et al. 2019, van der Tol et al. 2019, Han et al. 2020), photosynthetic electron flow distribution (Ye et al. 2013a,b), restraining backlight (Galle et al. 2011, Reynolds et al. 2012), and light protection (Murchie and Niyogi

\section{Highlights}

- The Freundlich model was applied to the analysis of grape photosynthesis data

- Five new photosynthetic parameters were obtained: maximum utilization of light energy $\left(\mathrm{LUE}_{\max }\right)$, light energy utilization index, efficient light intensity, falling velocity of LUE, rising velocity of LUE

- Oblique single cordon vine along the ditch performed better than traditional single cordon vine
Received 23 August 2020

Accepted 1 February 2021

Published online 25 February 2021

\author{
${ }^{+}$Corresponding authors \\ e-mail: 454691627@qq.com (X.Y.Wu) \\ panmq3399@sohu.com (M.Q.Pan)
}

\footnotetext{
Abbreviations: AQY - apparent quantum yield; DAP - daily accumulation of photosynthesis; $I_{\mathrm{c}}$ - light-compensation point; $I_{\mathrm{m}}$ - saturated light intensity; IQE - intrinsic quantum efficiency; LAR - light-adaptation range; LUE - light-use efficiency; LUE ${ }_{\max }-$ maximum utilization of light energy; LUI - light energy utilization index; OSC - oblique single cordon vine along the ditch; $\mathrm{PAR}_{\mathrm{me}}-$ efficient light intensity; $P_{\mathrm{N}}-$ net photosynthetic rate; $P_{\mathrm{Nmax}}-$ light-saturated net photosynthetic rate; $P_{\mathrm{Nmin}}-$ minimum net photosynthetic rate; $P_{\mathrm{Nsista}}$ - photosynthetic siesta value; $R_{\mathrm{D}}$ - respiration rate; TSC - traditional single cordon vine; $y_{\mathrm{f}}^{\prime}-$ falling velocity of LUE; $y_{\mathrm{r}}^{\prime}-$ rising velocity of LUE.

Acknowledgments: This study was supported by the China Agriculture Research System (CARS-29-ZP-8) and National Key R\&D Program of China (2018YFD0201309).

Conflict of interest: The authors declare that they have no conflict of interest.

These authors contributed equally to this work.
} 
2011, Reynolds et al. 2012). The mathematical models applied are mainly binomial regression (Zhang et al. 2011), least squares method (Meacham-Hensold et al. 2019), index model (Rascher et al. 2000), rectangular hyperbola model (Dias-Filho 2002), Yezipiao's model (Yezip) (Ye et al. 2013b), the right angle hyperbolic model (Ye et al. 2013a), dynamic model (Broadley et al. 2001), and the double exponential decay model (Han et al. 2005); some scholars also study diurnal change of photosynthesis using a Gaussian multi-peak model (Gauss) (Li et al. 2010). The extended Freundlich's model (FreundlichEXT) (Na 2020, San Martín et al. 2020) is often used to study physical adsorption. Some scholars have applied FreundlichEXT to the study of changes in the cement reaction rate ( $\mathrm{Li}$ and Dong 2013) but it has not been applied to the study of plant photosynthesis yet. In this study, we investigated photosynthesis in grape leaves in the southern margin of the Junggar basin in Xinjiang, China. Specifically, we focused on model fitting of photosynthesis-related indicators, using Gauss (Ye et al. 2013b) fitting of diurnal change of photosynthesis; Yezipiao's model (Yezip) (Harley et al. 1992) fitting for the light-response of photosynthetic rate; and FreundlichEXT model fitting for the response of light-use efficiency (LUE) to light intensity. LUE is the ratio of net photosynthetic rate to light intensity.

The southern margin of the Junggar basin in Xinjiang, China, has a mid-temperate climate. High temperatures above $35^{\circ} \mathrm{C}$ occur for $10-20 \mathrm{~d}$ per year in Changji prefecture on the southern margin of the Junggar basin, and the average temperature in the last 55 years has risen at a rate of $0.332^{\circ} \mathrm{C}$ per 10 years, making this one of the regions with the largest temperature increase in northern Xinjiang (Tan et al. 2018). The annual sunshine duration was about $2,850 \mathrm{~h}$, and the highest photosynthetic effective radiation intensity reached 2,600-2,900 $\mu$ mol(photon) $\mathrm{m}^{-2} \mathrm{~s}^{-1}$. The viticultural area is about 1,890 ha in this region, and grape production is usually dominated by horizontal or sloping trellises, with multiple, dispersed main tendrils or erect vines with a single trunk. The common features are a staggered arrangement of the new branches in each season, spatial overlap, inconsistent micro-environmental conditions of leaf and fruit growth, and poor light environment, which makes difficult to fundamentally improve the quality of grapes. Changing the trellis, cordon shape can affect the photosynthetic performance of leaves by improving the direction of nutrient supply, photosynthetic environment, Chl content, and chloroplast structure (Zhang et al. 2015, Majeed et al. 2020).

Through observation and empirical evidence, our team proposed that the optimal grape horizontal trellis in this region was 'oblique single cordon vine along the ditch' (OSC) (Zhang et al. 2015, Pan et al. 2017). The aboveground biomass was $22 \%$ higher than that of the traditional single cordon vine (TSC), the fruit quality was uniform, the maturity period was $5-7 \mathrm{~d}$ earlier, and the actual area of light energy captured was larger than that of the TSC (Zhang et al. 2015). We determined two photosynthetic parameters in this study, based on models to fit the measured data to predict potential photosynthetic indexes. These parameters were verified empirically, to compare their differences in the OSC and TSC leaves in photosynthesis. We also verified the role of several models including Gauss, Yezip, and FreundlichEXT in grape photosynthesis. These models revealed differences in photosynthesis of OSC and TSC leaves, and these results can be used to further optimize photosynthesis in grape leaves.

\section{Materials and methods}

Test materials and test conditions: The experiment was conducted in 2012-2016, Xinjiang, Urumqi city, Anningqu town, and in the Xinjiang Production and Construction corps sixth division 101 regiment. These areas are in the southern margin of the Junggar basin. The test materials were 'Red Globe' and 'Flame Seedless' grapes (Vitis vinifera L.), and the soil and irrigation conditions were modified to create the oblique single cordon vine along the ditch (OSC), as shown in Fig. 1. In both places, the traditional single cordon vine (TSC) was used as the control. The horizontal frame was used in the shape of both trees. The base of the TSC vines is erect, it is horizontally bound perpendicular to the planting row on the top of the frame, branches are crossed, and clusters are scattered. The vines of OSC tilt along the direction of the cultivation row and continue to be horizontally bound along the direction of the cultivation row after being put on the shelf. The branches are parallel, and the clusters are distributed in two strips.
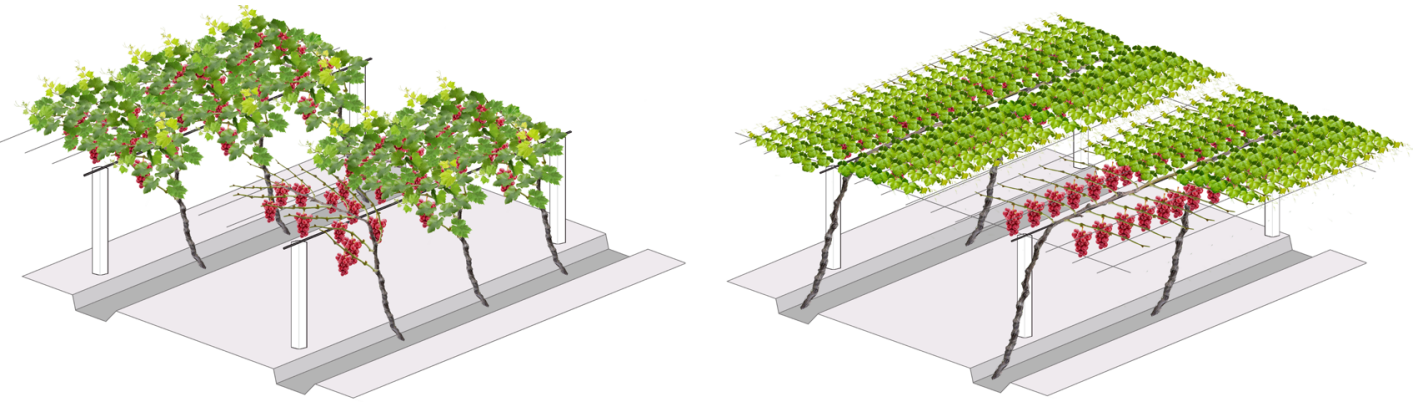

Fig. 1. Schematic diagram of traditional single cordon vine (TSC, on the left) and oblique single cordon vine along the ditch (OSC, on the right). 
Diurnal variation of photosynthetic parameters: In the fruit enlargement period (early July), which is typically sunny and breezy with natural light, we chose three similar OSC vines, with no plant diseases or insect pests. From these vines, we then chose three new leaves opposite the grape above the trellis. The CIRAS-2 photosynthetic system (PP-Systems, Amesbury, MA, USA) was used to determine photosynthetic parameters. Measurements were taken every $2 \mathrm{~h}$ from 09:00 to 19:00 h. According to the stability of the net photosynthetic rate $\left(P_{\mathrm{N}}\right)$ and $\mathrm{CO}_{2}$ difference, the measurement of each leaf lasted about 2 min. During the gas-exchange measurements, the leaf temperature was $22-32^{\circ} \mathrm{C}$, the water vapor pressure was 1,600-2,800 Pa, and the indoor $\mathrm{CO}_{2}$ concentration was $360-440 \mu \mathrm{mol}\left(\mathrm{CO}_{2}\right) \mathrm{mol}^{-1}$.

Photosynthesis response to light intensity: From 09:30 11:30 h, the PAR was controlled by the system's own light source, and 11 gradient steps of $0-2,650 \mu \mathrm{mol}$ (photon) $\mathrm{m}^{-2} \mathrm{~s}^{-1}$ were set in reverse order $[2,650 ; 2,031 ; 1,560$; 938, 557, 400, 293, 220, 117, 37, $0 \mu \mathrm{mol}$ (photon) $\mathrm{m}^{-2} \mathrm{~s}^{-1}$. Leaf selection was the same as for the measurement of diurnal variation of photosynthetic parameters. During the gas-exchange measurements, the leaf temperature was $28 \pm 0.5^{\circ} \mathrm{C}$, the indoor $\mathrm{CO}_{2}$ concentration was $400 \pm 10$ $\mu \mathrm{mol}\left(\mathrm{CO}_{2}\right) \mathrm{mol}^{-1}$, and the water vapor pressure was 2,200 2,500 Pa.

Target blade quality and environmental control: In order to ensure the reliability of the test results, the days of photosynthetic measurement in both places were close to each other and the weather conditions were similar. Both were sunny and breezy, with the lowest temperature of $20^{\circ} \mathrm{C}$ and the highest temperature of $31^{\circ} \mathrm{C}$. The selection of target leaves excluded the influence of tree body and branch nutrition, leaf position, leaf age, and other factors. There were no significant differences in total $\mathrm{Chl}$ content, leaf temperature, PAR, atmospheric vapor pressure or $\mathrm{CO}_{2}$ concentration under the same treatment between the target leaves.

Diurnal variation characteristics of photosynthetic rate based on Gaussian multi-peak model (Gauss): The Gauss model (Li et al. 2010) can be expressed as follows:

$$
y=y_{0}+\frac{A_{1}}{w_{1} \sqrt{\pi / 2}} e^{-2\left(\frac{x-x_{1}}{w_{1}}\right)^{2}}+\frac{A_{2}}{w_{2} \sqrt{\pi / 2}} e^{-2\left(\frac{x-x_{2}}{w_{2}}\right)^{2}}
$$

where $y$ is the net photosynthetic rate $\left(P_{\mathrm{N}}\right)\left[\mu \mathrm{mol} \mathrm{m} \mathrm{m}^{-2} \mathrm{~s}^{-1}\right.$; $x$ is time $[\mathrm{h}] ; y_{0}$ is the baseline; $\mathrm{A}_{1}$ and $\mathrm{A}_{2}$ are the integral total area on the baseline under the two peak curves; $w_{1}$ and $w_{2}$ are the half height and width of the two peaks, respectively; and $x_{1}$ and $x_{2}$ are the peak values of the two peaks, respectively. Gauss was fitted by Origin 2018 to calculate the full width at half maximum (FWHM) and the peak net photosynthetic rate $y_{\mathrm{c}}\left[\mu \mathrm{mol} \mathrm{m} \mathrm{m}^{-2} \mathrm{~s}^{-1}\right]$.

$$
\begin{aligned}
& \mathrm{FWHM}=w \times \sqrt{\ln (4)} \\
& y_{\mathrm{c}}=y_{0}+\frac{A}{w \sqrt{\pi / 2}}
\end{aligned}
$$

Light-response characteristics analysis of photosynthetic rate based on the Yezipiao's model (Yezip): The expression of Yezip (Ye et al. 2013b) is as follows:

$P_{\mathrm{N}}(I)=\alpha \frac{1-\beta I}{1+\gamma I} I-R_{\mathrm{D}}$

where $\alpha, \beta$, and $\gamma$ are three coefficients, respectively, initial quantum efficiency (IQE), photoinhibition [ $\mu$ mol(photon) $\mathrm{m}^{-2} \mathrm{~s}^{-1}$, and saturated light intensity $\left[\mu \mathrm{mol}\right.$ (photon) $\left.\mathrm{m}^{-2} \mathrm{~s}^{-1}\right]$; $I$ is the photosynthetic effective radiation intensity (PAR) [ $\mu \mathrm{mol}\left(\right.$ photon) $\mathrm{m}^{-2} \mathrm{~s}^{-1}$ ], and $R_{\mathrm{D}}$ is the dark respiration rate $\left[\mu \mathrm{mol} \mathrm{m} \mathrm{m}^{-2} \mathrm{~s}^{-1}\right]$. Origin 2018 was used to fit the right-angle hyperbolic correction model for the light-response of net photosynthetic rate. The maximum net photosynthetic rate $\left(P_{\mathrm{Nmax}}\right)\left[\mu \mathrm{mol} \mathrm{m} \mathrm{m}^{-2} \mathrm{~s}^{-1}\right]$, dark respiration rate $\left(R_{\mathrm{D}}\right)[\mu \mathrm{mol}$ $\left.\mathrm{m}^{-2} \mathrm{~s}^{-1}\right]$, saturated light intensity $\left(I_{\mathrm{m}}\right)\left[\mu \mathrm{mol}\right.$ (photon) $\left.\mathrm{m}^{-2} \mathrm{~s}^{-1}\right]$, light-compensation point $\left(I_{\mathrm{c}}\right)\left[\mu \mathrm{mol}\left(\right.\right.$ photon) $\mathrm{m}^{-2} \mathrm{~s}^{-1}$, intrinsic quantum efficiency $\left(\mathrm{IQE}_{I_{\mathrm{o}}}\right)$ and apparent quantum efficiency at the light-compensation point $\left(\mathrm{AQE}_{I \mathrm{c}}\right)$ were calculated as follows:

$$
\begin{aligned}
& I_{\mathrm{m}}=\frac{\sqrt{(\beta+\gamma) / \beta}-1}{\gamma} \\
& P_{\mathrm{Nmax}}=\alpha \frac{\sqrt{\beta+\gamma}-\sqrt{\beta}^{2}}{\gamma}-R_{\mathrm{D}} \\
& I_{\mathrm{c}}=\frac{\alpha-\gamma R_{\mathrm{D}}-\sqrt{\left.\gamma R_{\mathrm{D}}-\alpha\right)^{2}-4 \alpha \beta R_{\mathrm{D}}}}{2 \alpha \beta} \\
& \mathrm{IQE}_{I_{0}}=\alpha\left[1+(\gamma+\beta) I_{\mathrm{c}}\right] \\
& \mathrm{AQY}_{I_{\mathrm{c}}}=\alpha \frac{1+(\gamma-\beta) I_{\mathrm{c}}-\beta \gamma I_{\mathrm{c}}{ }^{2}}{\left(1+\gamma I_{\mathrm{c}}\right)^{2}}
\end{aligned}
$$

Analysis of the characteristics of the light response based on the extended Freundlich's model (FreundlichEXT): According to the development and changes in the relationship curve between the LUE and the photosynthetic effective radiation intensity, the FreundlichEXT was in good agreement with the curve. Therefore, the FreundlichEXT was used to calculate the photosynthetic parameters of reference values to evaluate the LUE of plants. The FreundlichEXT model (Li and Dong 2013) is expressed as follows:

$y=a x^{b x^{-c}}$

where $y$ is light-use efficiency (LUE) and $x$ is photosynthetic effective radiation intensity (PAR) $\left[\mu \mathrm{mol}\right.$ (photon) $\left.\mathrm{m}^{-2} \mathrm{~s}^{-1}\right]$. Origin 2018 was used to fit the FreundlichEXT model, and calculate maximum light energy utilization LUE, and efficient light intensity $\mathrm{PAR}_{\max }\left[\mu \mathrm{mol}\left(\right.\right.$ photon) $\left.\mathrm{m}^{-2} \mathrm{~s}^{-1}\right]$ (light intensity at maximum LUE); LUE rising rate and falling rate (with maximum LUE as the bound, the slope of the rising and falling LUE data is calculated by linear fitting), and light energy utilization index [LUI, the sum 
of the absolute value of the slope $y^{\prime}$ of the light energy utilization ratio at saturated light intensity $\left(I_{\mathrm{m}}\right)$ and lightcompensation point $\left.\left(I_{\mathrm{c}}\right)\right]$.

$$
\begin{aligned}
& \text { LUEmax }_{\text {max }}=e^{\frac{1}{\mathrm{c}}} \\
& \text { PAR }_{\text {efficient }}=\mathrm{a} \times e^{\frac{\mathrm{b}}{\mathrm{c} \times \mathrm{e}}} \\
& y^{\prime}=\mathrm{ab} x^{\left(-1-\mathrm{c}+\mathrm{b} x^{-\mathrm{c}}\right)(1-\mathrm{c} \ln [x])}
\end{aligned}
$$

\section{Results}

Diurnal variation characteristics of photosynthetic rate based on the Gauss model: The Gauss model in Origin 2018 was used to approximate the diurnal change of net photosynthetic rate for OSC 'Red Globe', TSC 'Red Globe', OSC 'Flame Seedless', and TSC 'Flame Seedless' (Fig. 2). $R^{2}$ correlation coefficients were, respectively, $0.9787,0.9842,0.98961$, and 0.9888 . Fitting equations are shown in Appendix, and fitting parameters are shown in Table 1. Through Gauss, parameters, such as $y_{0}, x_{1}, x_{2}$, $w_{1}, w_{2}$, FWHM peak, and $y_{\mathrm{c}}$ can be calculated: $y_{0}$ is the minimum net photosynthetic rate that can be effectively simulated by this model, and $y_{0}$ of the two varieties grapes under the OSC shape was larger than that of the TSC shape. The $x_{1}$ and $x_{2}$ are the times when the main and the second peak of the photosynthetic rate appeared. The time when the diurnal peak of photosynthetic rate appeared was different between the two varieties of grapes. The peak of photosynthetic rate of 'Red Globe' grape appeared slightly later than that of 'Flame Seedless' grape, and the main peak of photosynthetic rate of OSC appeared earlier than that of TSC.

The $w_{1}$, and $w_{2}$ are light-adaptive parameters, which reflect grape leaf photosynthetic response speed of the photosynthetic environment. The greater the $w$ value, the faster the net photosynthetic rate changes, and vice versa.
In this study, the light adaptation duration of 'Red Globe' grape was longer than that of 'Flame Seedless' grape and OSC was longer than that of TSC.

According to Li et al. (2010), for Gauss fitting in the process of diurnal change of photosynthetic rate, $A_{1}$ and $A_{2}$ can reflect the size of the plant photosynthetic rate, and the size of the accumulated value can reflect the size of the photosynthetic product accumulation amount in theory. In this study, $A_{1}+A_{2}$ were greater in 'Flame Seedless' than in 'Red Globe' grapes, and OSC was greater than that of the TSC.

The FWHM reflects the photosynthetic adaptation range of grape leaves to the photosynthetic environment, within which the leaves are well adapted to the photosynthetic environment. With time, the photosynthesis environment changes, and the longer the duration, the stronger the photosynthetic ability to adapt. $\mathrm{FWHM}_{1}$ embodies the light in the morning from weak to strong, in the process of temperature changing from low to high, light intensity on leaves, and temperature adaptability. $\mathrm{FWHM}_{2}$ is the adaptation ability of leaves to the change of light intensity and temperature during the change of afternoon light intensity from strong to weak and temperature from high to low. In this study, the 'Red Globe' FWHM was larger than that of 'Flame Seedless', and the FWHM of both OSC grape varieties was larger than of the TSC.

The $y_{\mathrm{c}}$ is the peak of net photosynthetic rate, i.e., the maximum net photosynthetic rate. The $y_{\mathrm{c}}$ of 'Flame Seedless' leaves was higher than that of 'Red Globe', and the OSC of 'Red Globe' was higher than that of TSC.

Light-response characteristics analysis of photosynthetic rate based on the Yezip model: The response characteristics of photosynthetic rate to light intensity of grape leaves were fitted with the Yezip model, and the actual measured values were distributed near the curve or coincident with the curve (Fig. 3). The correlation coefficients $\left(R^{2}\right)$ were 0.9042 and 0.9092 for OSC and TSC

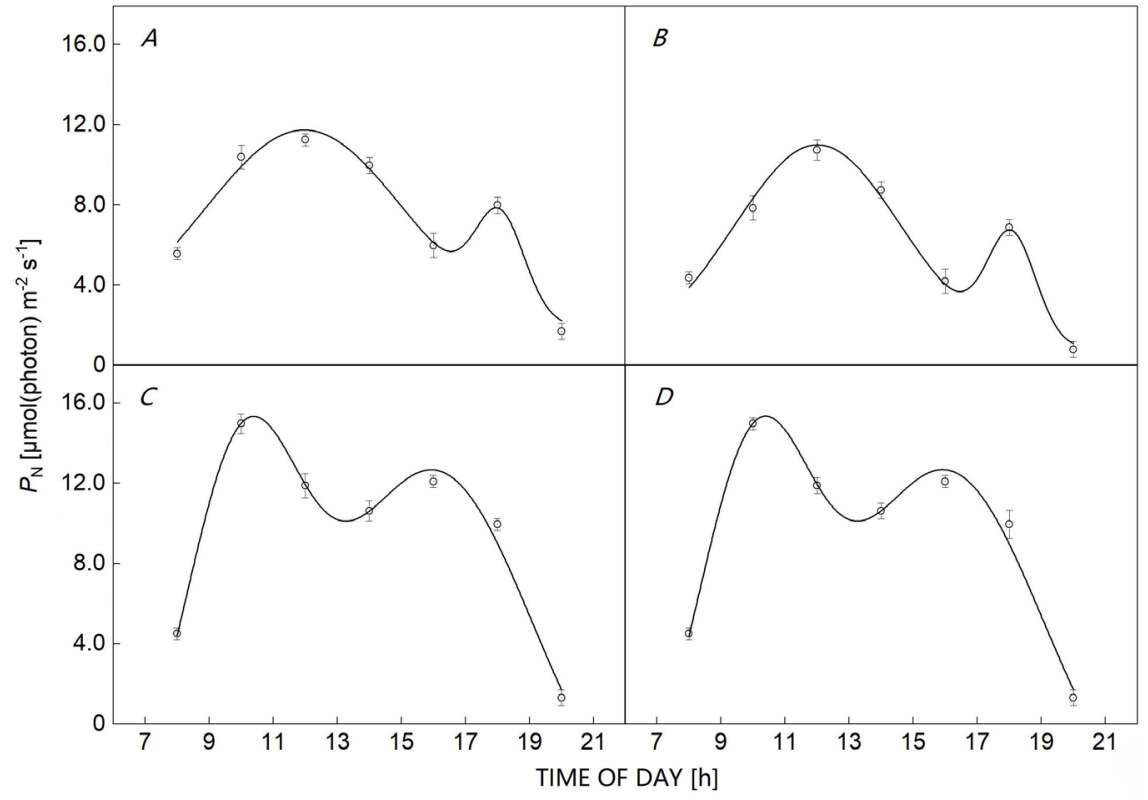

Fig. 2. The diurnal variation curve of net photosynthetic rate was fitted by Gauss model. $(A)$ 'Red Globe' OSC; (B) 'Red Globe' TSC; (C) 'Flame Seedless' OSC; $(D)$ 'Flame Seedless' TSC. OSC - oblique single cordon vine along the ditch; TSC - traditional single cordon vine. The open circles represent the average value, the error bars represent $95 \%$ and $5 \%$ confidence intervals, respectively. 


\section{F.C. ZHANG et al.}

Table 1. Multi-peak fitting parameters of diurnal variations of photosynthesis. OSC - oblique single cordon vine along the ditch; $P_{\mathrm{Nmax}}-$ net photosynthetic rate peak; $P_{\mathrm{Nmin}}-$ minimum net photosynthetic rate; $P_{\mathrm{Nsiesta}}-$ photosynthetic siesta value; TSC - traditional single cordon vine. The values are means \pm SD. The means in the columns within different parameters followed by different letters are significantly different (LSD test, $P<0.01)$.

\begin{tabular}{|c|c|c|c|c|c|c|}
\hline \multirow[t]{2}{*}{ Peaks/Valley } & \multirow[t]{2}{*}{ Parameter } & \multirow[t]{2}{*}{ Parameter meaning } & \multicolumn{2}{|l|}{ 'Red Globe' } & \multicolumn{2}{|c|}{ 'Flame Seedless' } \\
\hline & & & OSC & $\mathrm{TSC}$ & OSC & $\mathrm{TSC}$ \\
\hline \multirow{3}{*}{ Main peak } & $y_{0}$ & 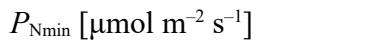 & $1.70 \pm 0.20^{\mathrm{A}}$ & $0.81 \pm 0.31^{\mathrm{B}}$ & $1.30 \pm 0.32^{\mathrm{A}}$ & $0.53 \pm 0.21^{\mathrm{B}}$ \\
\hline & $x_{1}$ & Peak time & $11.97 \pm 0.14^{\mathrm{A}}$ & $12.02 \pm 0.32^{\mathrm{A}}$ & $9.93 \pm 0.31^{\mathrm{A}}$ & $10.26 \pm 0.21^{\mathrm{A}}$ \\
\hline & $w_{1}$ & Parameter of light adaptation & $6.22 \pm 0.82^{\mathrm{A}}$ & $5.18 \pm 0.60^{\mathrm{B}}$ & $3.84 \pm 0.40^{\mathrm{A}}$ & $3.40 \pm 0.42^{\mathrm{A}}$ \\
\hline \multirow{10}{*}{$\begin{array}{l}\text { Secondary } \\
\text { peak }\end{array}$} & $A_{1}$ & Integral area of peak & $78.26 \pm 8.22^{\mathrm{A}}$ & $66.20 \pm 8.70^{\mathrm{B}}$ & $62.95 \pm 6.11^{\mathrm{A}}$ & $58.95 \pm 6.43^{\mathrm{A}}$ \\
\hline & $\operatorname{sigma}_{1}$ & Capability sigma & $3.11 \pm 0.22^{\mathrm{A}}$ & $2.59 \pm 0.22^{\mathrm{A}}$ & $1.92 \pm 0.12^{\mathrm{A}}$ & $1.70 \pm 0.10^{\mathrm{A}}$ \\
\hline & $\mathrm{FWHM}_{1}$ & Full width at half maximum & $7.33 \pm 0.91^{\mathrm{A}}$ & $6.10 \pm 0.83^{\mathrm{B}}$ & $4.52 \pm 0.43^{\mathrm{A}}$ & $4.00 \pm 0.40^{\mathrm{A}}$ \\
\hline & $y_{\mathrm{c} 1}$ & $P_{\mathrm{N} \max }\left[\mu \mathrm{mol} \mathrm{m} \mathrm{m}^{-2} \mathrm{~s}^{-1}\right]$ & $11.74 \pm 0.83^{\mathrm{A}}$ & $11.00 \pm 1.02^{\mathrm{B}}$ & $14.38 \pm 0.82^{\mathrm{A}}$ & $14.36 \pm 1.13^{\mathrm{A}}$ \\
\hline & $x_{2}$ & Peak time & $18.08 \pm 0.62^{\mathrm{A}}$ & $18.07 \pm 0.53^{\mathrm{A}}$ & $16.09 \pm 0.43^{\mathrm{A}}$ & $15.91 \pm 0.52^{\mathrm{A}}$ \\
\hline & $w_{2}$ & Parameter of light adaptation & $1.51 \pm 0.24^{\mathrm{A}}$ & $1.51 \pm 0.24^{\mathrm{A}}$ & $5.23 \pm 1.11^{\mathrm{A}}$ & $4.80 \pm 1.12^{\mathrm{B}}$ \\
\hline & $A_{2}$ & Integral area of peak & $8.78 \pm 1.22^{\mathrm{B}}$ & $9.95 \pm 1.42^{\mathrm{A}}$ & $69.91 \pm 5.12^{\mathrm{A}}$ & $69.82 \pm 6.90^{\mathrm{A}}$ \\
\hline & $\operatorname{sigma}_{2}$ & Capability sigma & $0.75 \pm 0.11^{\mathrm{A}}$ & $0.75 \pm 0.11^{\mathrm{A}}$ & $2.61 \pm 0.31^{\mathrm{A}}$ & $2.40 \pm 0.33^{\mathrm{A}}$ \\
\hline & $\mathrm{FWHM}_{2}$ & Full width at half maximum & $1.78 \pm 0.12^{\mathrm{A}}$ & $1.78 \pm 0.20^{\mathrm{A}}$ & $6.15 \pm 0.50^{\mathrm{A}}$ & $5.65 \pm 0.42^{\mathrm{B}}$ \\
\hline & $y_{\mathrm{c} 2}$ & 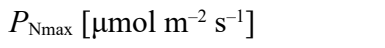 & $6.34 \pm 0.61^{\mathrm{A}}$ & $6.07 \pm 0.70^{\mathrm{A}}$ & $11.97 \pm 0.81^{\mathrm{A}}$ & $12.14 \pm 1.01^{\mathrm{A}}$ \\
\hline Valley & $y_{0}{ }^{\prime}$ & 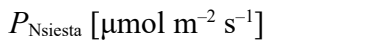 & $5.70 \pm 0.41^{\mathrm{A}}$ & $3.70 \pm 0.51^{\mathrm{B}}$ & $10.10 \pm 1.12^{\mathrm{A}}$ & $10.10 \pm 1.42^{\mathrm{A}}$ \\
\hline
\end{tabular}

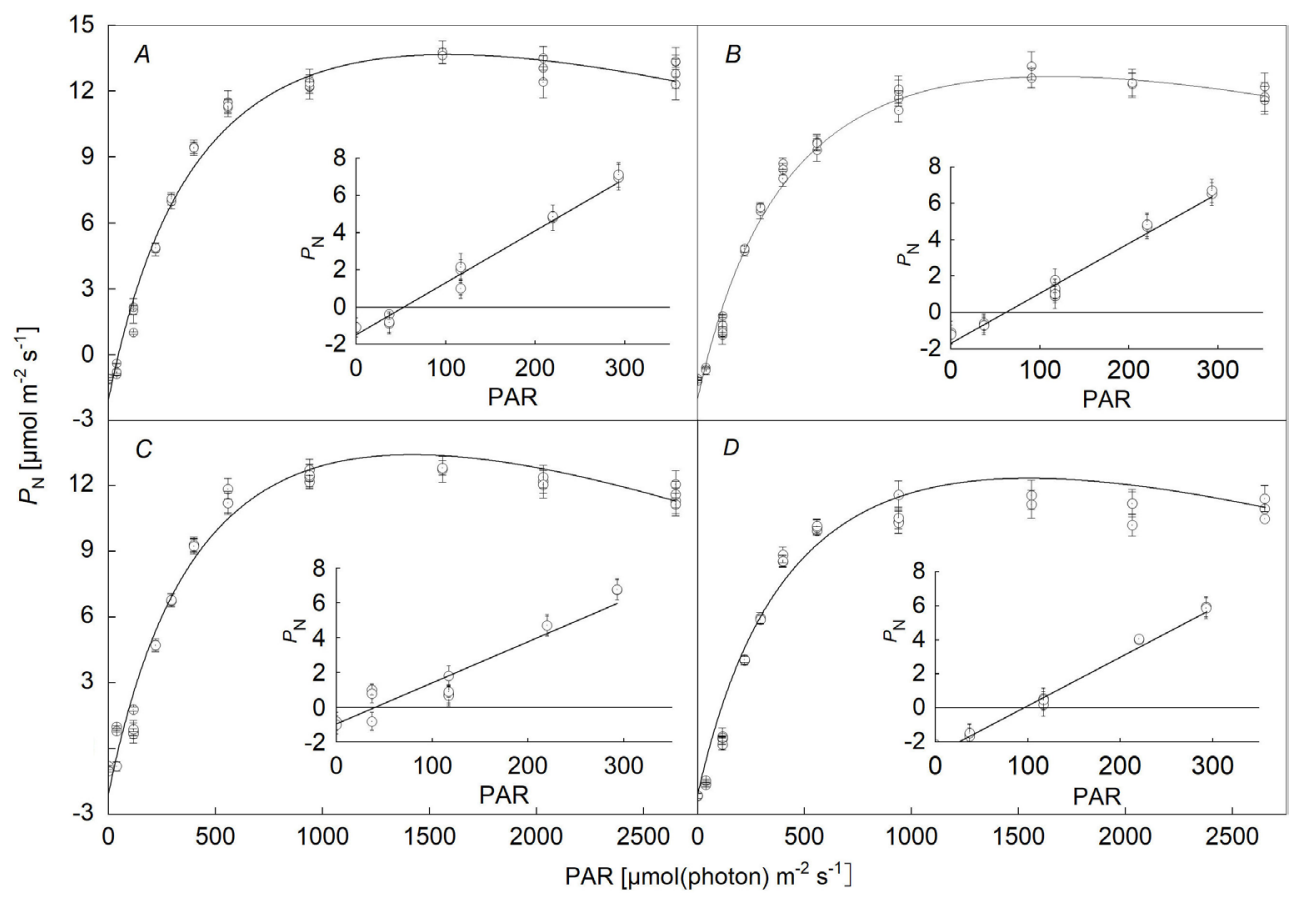

Fig. 3. Curve of photosynthetic rate response to PAR intensity fitted by Yezip model and linear fitting of net photosynthetic rate under low light. The open circles represent the average value, the error bars represent $95 \%$ and $5 \%$ confidence intervals, respectively. ( $A$ ) 'Red Globe' OSC; $(B)$ 'Red Globe' TSC; $(C)$ 'Flame Seedless' OSC; $(D)$ 'Flame Seedless' TSC. OSC - oblique single cordon vine along the ditch; PAR - photosynthetically active radiation; $P_{\mathrm{N}}-$ net photosynthetic rate; TSC - traditional single cordon vine.

'Red Globe', and 0.8950 and 0.8997 for OSC and TSC 'Flame Seedless', respectively. The fitting equations are shown in Appendix.

Saturated light intensity $\left(I_{\mathrm{m}}\right)$, light-compensation point $\left(I_{c}\right)$, and light-adaptation range (LAR) were calculated. The results showed that the photosynthetic utilization range of leaves of the two varieties with OSC shape was larger than that for TSC shape, and the saturation light intensity $I_{\mathrm{m}}$ was higher than that for TSC shape. The light-compensation point of 'Red Globe' and 'Flame Seedless' grape leaves 
was $28 \mu$ mol(photon) $\mathrm{m}^{-2} \mathrm{~s}^{-1}$. The calculated maximum photosynthetic rate $\left(P_{\mathrm{N} \max }\right)$ of the OSC was better than that of TSC, and $P_{\mathrm{Nmax}}$ of the 'Flame Seedless' vine was bigger than that of 'Red Globe'. The apparent quantum efficiency and intrinsic quantum efficiency at the light-compensation point of the leaves of the OSC were both higher than that of the TSC, and the 'Red Globe' was slightly lower than that of 'Flame Seedless'. The dark respiration rate $\left(R_{\mathrm{D}}\right)$ was smaller than the TSC, and the 'Red Globe' was smaller than the 'Flame Seedless' (Fig. 4).

Response characteristic analysis of LUE to PAR intensity based on the FreundlichEXT model: The FreundlichEXT model was used to fit the response of light energy utilization rate to photosynthetic effective radiation intensity (Fig. 5). The four $R^{2}$ values were 0.9833 ('Red
Globe' OSC), 0.9551 ('Red Globe' TSC), 0.9474 ('Flame Seedless' OSC), and 0.9253 ('Flame Seedless' TSC). The fitting equation is shown in Appendix, and the fitting parameters are shown in Table 2.

With the increase of photosynthetic effective radiation intensity, the LUE increased first and then decreased. The LUE of 'Red Globe' grape leaves rose rapidly from 0-271 umol(photon) $\mathrm{m}^{-2} \mathrm{~s}^{-1}$, and then began to decline. The leaf LUE of 'Flame Seedless' OSC and TSC rose rapidly from 0 to $373 \mu \mathrm{mol}$ (photon) $\mathrm{m}^{-2} \mathrm{~s}^{-1}$, and then began to decline. The LUE of TSC leaves increased rapidly from 0-296 $\mu \mathrm{mol}$ (photon) $\mathrm{m}^{-2} \mathrm{~s}^{-1}$ and then began to decrease. The LUE of OSC was higher than that of the TSC. The descending rates of the four treatments showed a trend of fast and then slow decreases. The maximum light energy utilization ratio of 'Red Globe' leaves was higher than that

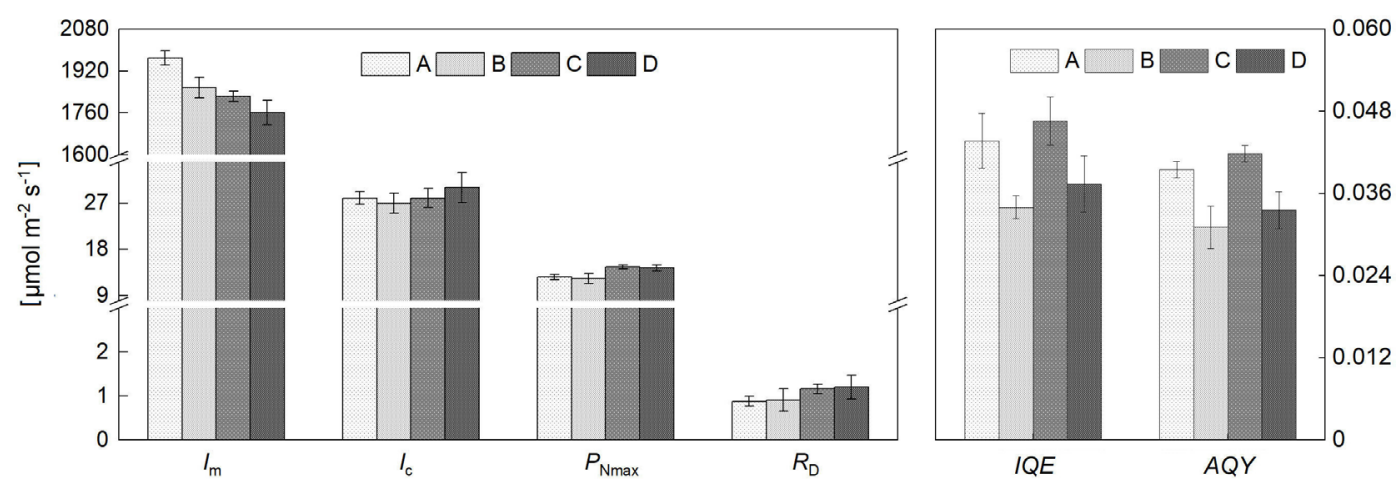

Fig. 4. Parameters of photosynthetic response to light fit by the rectangular hyperbolic correction model. AQY - apparent quantum yield; $I_{\mathrm{c}}$ - light-compensation point; $I_{\mathrm{m}}$ - saturated light intensity; IQE - intrinsic quantum efficiency; $P_{\mathrm{Nmax}}-$ light-saturated net photosynthetic rate; $R_{\mathrm{D}}$ - respiration rate. The error bars represent $95 \%$ and $5 \%$ confidence intervals, respectively. A - 'Red Globe' OSC; B - 'Red Globe' TSC; C - 'Flame Seedless' OSC; D - 'Flame Seedless' TSC. OSC - oblique single cordon vine along the ditch; TSC - traditional single cordon vine.

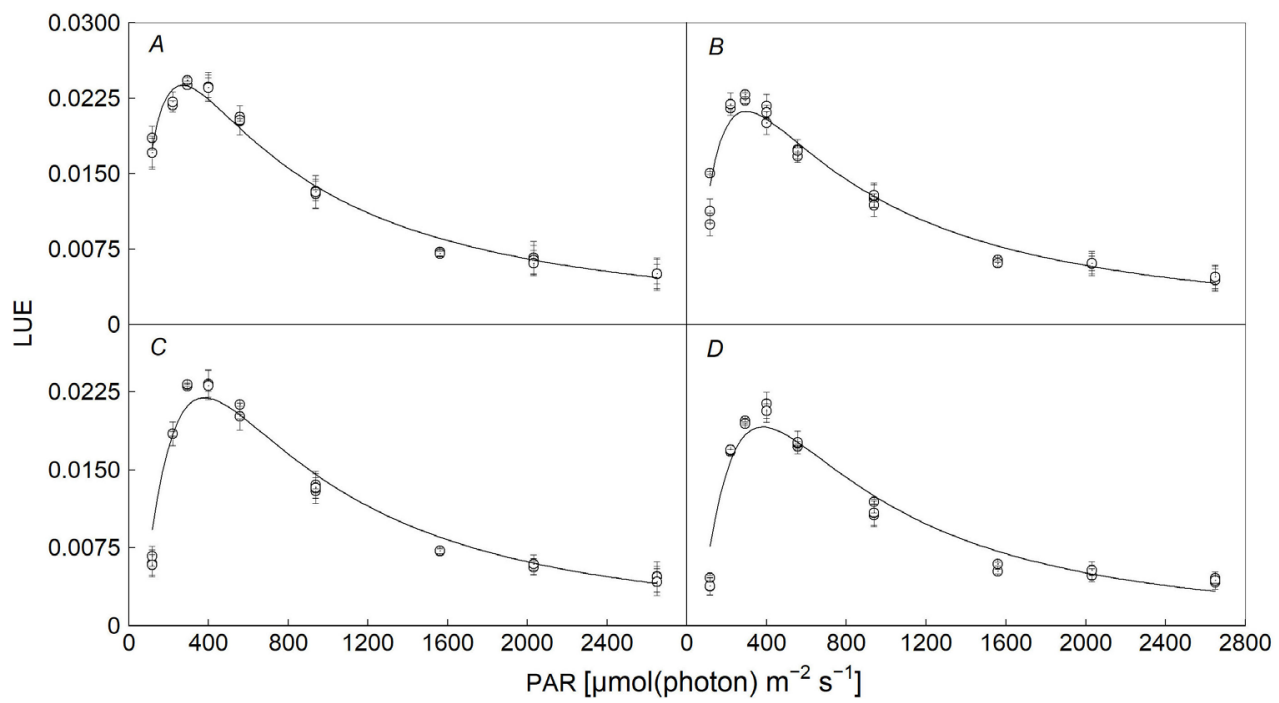

Fig. 5. Response characteristics analysis of light-use efficiency (LUE) to PAR intensity fitted by FreundlichEXT model. PAR photosynthetically active radiation. The open circles represent the average value, the error bars represent $95 \%$ and $5 \%$ confidence intervals, respectively. $(A)$ 'Red Globe' OSC; $(B)$ 'Red Globe' TSC; $(C)$ 'Flame Seedless' OSC; $(D)$ 'Flame Seedless' TSC. OSC oblique single cordon vine along the ditch; TSC - traditional single cordon vine. 


\section{F.C. ZHANG et al.}

of 'Flame Seedless', and the OSC was higher than that of the TSC. The PAR producing the maximum light energy utilization ratio of 'Red Globe' leaves was lower than that of 'Flame Seedless', the LUE was higher than that of 'Flame Seedless', and the OSC was higher than that of the TSC (Figs. 5, 6; Table 1).

\section{Verification and evaluation of the physical meaning of} the model: Previous studies have verified and evaluated the physical meaning of the Gauss (Li et al. 2010) and Yezip (Ye et al. 2013b) models, thus, this paper only verifies and evaluates the physical meaning of the FreundlichEXT model.

(1) Maximum utilization of light energy $\left(\mathrm{LUE}_{\max }\right)$. The LUE of plants is different under different PAR intensities. It is thus necessary to study plants under different PAR intensities during changes of LUE, to determine the maximum utilization of light energy (LUE $\mathrm{Lax}_{\max }$ ). In Fig. 4,

Table 2. Parameters of light energy-utilization ratio response to light intensity based on the Freundlich model. OSC - oblique single cordon vine along the ditch; $\mathrm{LUE}_{\max }$ - maximum utilization of light energy; LUI - light energy utilization index; $\mathrm{PAR}_{\mathrm{me}}-$ efficient light intensity $\left[\mu \mathrm{mol}\right.$ (photon) $\mathrm{m}^{-2} \mathrm{~s}^{-1}$; TSC - traditional single cordon vine; $y_{\mathrm{f}}^{\prime}$ - falling velocity of LUE; $y_{\mathrm{r}}^{\prime}-$ rising velocity of LUE.

\begin{tabular}{llllll}
\hline \multicolumn{2}{c}{$\begin{array}{l}\text { 'Red Globe' } \\
\text { OSC }\end{array}$} & \multicolumn{2}{l}{ TSC } & 'Flame Seedless' & OSC \\
\hline$y_{\mathrm{r}}^{\prime}$ & Fitting values & $3.21 \times 10^{-5} \pm 6.73 \times 10^{-6}$ & $3.91 \times 10^{-5} \pm 6.44 \times 10^{-6}$ & $4.23 \times 10^{-5} \pm 5.81 \times 10^{-6}$ & $3.95 \times 10^{-5} \pm 5.18 \times 10^{-6}$ \\
& Measured values & $3.74 \times 10^{-5} \pm 3.28 \times 10^{-6}$ & $6.37 \times 10^{-5} \pm 1.27 \times 10^{-5}$ & $5.82 \times 10^{-5} \pm 1.23 \times 10^{-5}$ & $5.51 \times 10^{-5} \pm 9.86 \times 10^{-6}$ \\
& Deviation [\%] & 14.21 & 10.51 & 12.07 & 12.61 \\
$y_{\mathrm{f}}^{\prime}$ & Fitting values & $7.54 \times 10^{-6} \pm 2.56 \times 10^{-7}$ & $7.04 \times 10^{-6} \pm 2.28 \times 10^{-7}$ & $7.83 \times 10^{-6} \pm 2.34 \times 10^{-7}$ & $7.04 \times 10^{-6} \pm 2.12 \times 10^{-7}$ \\
& Measured values & $8.04 \times 10^{-6} \pm 8.17 \times 10^{-7}$ & $6.96 \times 10^{-6} \pm 6.70 \times 10^{-7}$ & $7.19 \times 10^{-6} \pm 8.03 \times 10^{-7}$ & $6.33 \times 10^{-6} \pm 9.66 \times 10^{-7}$ \\
& Deviation [\%] & 6.235 & 1.121 & 8.913 & 11.05 \\
LUE $_{\text {max }}$ & Fitting values & 0.024 & 0.021 & 0.022 & 0.019 \\
& Measured values & 0.024 & 0.023 & 0.023 & 0.020 \\
& Deviation [\%] & 0.00 & 7.30 & 5.68 & 4.76 \\
PAR $_{\text {me }}$ & Fitting values & 271 & 296 & 373 & 373 \\
& Measured values & 275 & 300 & 400 & 400 \\
& Deviation [\%] & 1.45 & 1.33 & 6.75 & 6.75 \\
LUI & Fitting values & $5.63 \times 10^{-9}$ & $9.25 \times 10^{-10}$ & $3.11 \times 10^{-12}$ & $3.68 \times 10^{-13}$ \\
& Measured values & $5.63 \times 10^{-9}$ & $9.25 \times 10^{-10}$ & $3.11 \times 10^{-12}$ & $3.68 \times 10^{-13}$ \\
& Deviation [\%] & 0.00 & 0.00 & 0.00 & 0.00 \\
\hline
\end{tabular}

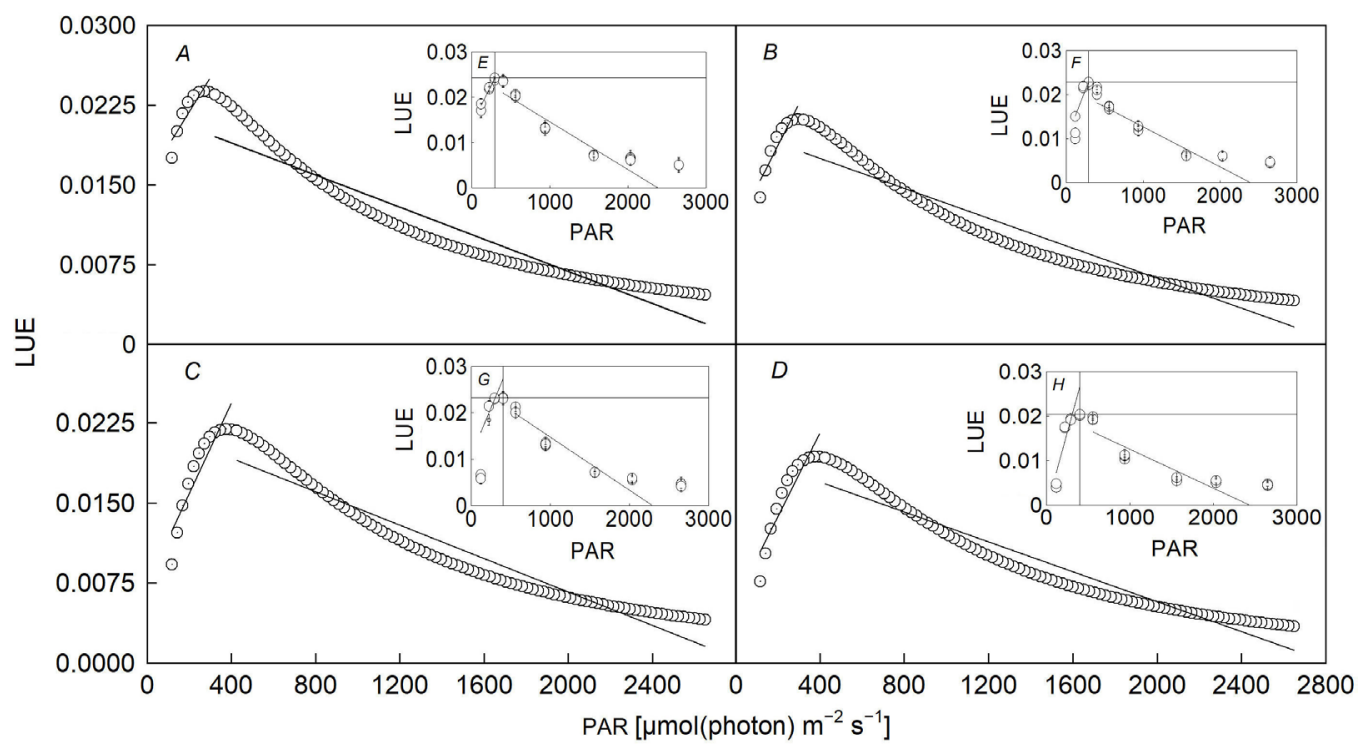

Fig. 6. The ascending and descending slopes of the response of light-use efficiency (LUE) to light intensity between Freundlich model fitting and measured. PAR - photosynthetically active radiation. The open circles represent the average value, the error bars represent 95\% and 5\% confidence intervals, respectively. $(A)$ 'Red Globe' OSC; $(B)$ 'Red Globe' TSC; $(C)$ 'Flame Seedless' OSC; $(D)$ 'Flame Seedless' TSC. OSC - oblique single cordon vine along the ditch; TSC - traditional single cordon vine. 
the $y$-coordinate of the highest point of the curve is $\mathrm{LUE}_{\max }$, and Eq. 11 is used to calculate the fitting value of $L_{U E}$ max. On the basis of optical response, LUE under each gradient PAR was calculated and $\mathrm{LUE}_{\max }$ was preliminarily screened out. According to the change rate of LUI, the corresponding PAR of $\mathrm{LUE}_{\max }$ was found. The PAR was then set between 250-400 $\mu$ mol(photon) $\mathrm{m}^{-2} \mathrm{~s}^{-1}$ to measure the optical response, calculate LUE, and determine the actual measured value of $\mathrm{LUE}_{\max }$. The error of the maximum fitting light energy utilization ratio was $\leq 7.3 \%$ compared with the measured value (Table 2 ).

(2) Efficient light intensity $\left(\mathrm{PAR}_{\mathrm{me}}\right)$. As described in (1), under different PAR, the LUE of the plant is different. Under a certain PAR, the LUE of the plant leaves reaches the maximum, and then the PAR is the efficient light intensity. The high-efficiency intensity is the $x$-coordinate of the highest point of the curve in Fig. 5. Through iterative calculation of the LUI, the fitting value was calculated using Eq. 12 to get the fitting value of $\mathrm{PAR}_{\mathrm{me}}$. The measurement method was the same as (1), and the LUE $\mathrm{max}_{\max }$ was obtained. The corresponding PAR is the 'measured value of PAR $\mathrm{P}_{\mathrm{me}}$ '. The error of fitting $\mathrm{PAR}_{\mathrm{me}}$ was less than $6.75 \%$ compared to the measured value (Table 2).

(3) Rate of increase $\left(y_{\mathrm{r}}^{\prime}\right)$ and rate of decrease $\left(y_{\mathrm{f}}^{\prime}\right)$ of LUE. In the actual photosynthesis process, these are the rates of increase in the LUE during the morning sunlight intensification process, and the rate of decline in the LUE during afternoon sunlight fade-out. Using the function of 'duplicate data segment' of the Origin 2018 fitting curve, the fitting data were proposed and a linear regression of the rising and falling trends was conducted with the vertex coordinates as the boundary, that is, the rising and falling rates of fitting light energy utilization. For the measured net photosynthetic rate, we calculated the LUE, and used the rising segment and the falling segment to establish a linear relationship with the photosynthetic effective radiation intensity (Fig. 5). This allowed us to obtain the measured values of the rising and falling rates of the light energy-utilization rate. The error of the fitting light energy utilization rate rise rate was $14.2 \%$, compared to the measured value (Table 2). The reason for the large error is that the value is calculated by measuring the net photosynthetic rate under the condition of weak light close to $0 \mu \mathrm{mol} \cdot \mathrm{m}^{-2} \cdot \mathrm{s}^{-1}$. In the actual measurement process, it is difficult to accurately measure the net photosynthetic rate under the conditions of the weakest light. The error of the fitting value of $y_{\mathrm{f}}^{\prime}$ was less than $8.9 \%$ compared to the measured value, except that the error of 'Flame Seedless' TSC treatment was 11.1.

(4) Index of light energy utilization (LUI). By fitting the relationship between net photosynthetic rate and photosynthetic effective radiation intensity, the lightcompensation point and saturated light intensity were calculated. The slope $y^{\prime}\left(I_{\mathrm{c}}\right)$ value of the light energyutilization rate at the light-compensation point and the slope $y^{\prime}\left(I_{c}\right)$ value of the light energy-utilization rate at the saturated light intensity were calculated using Eq. 13, and the sum of the absolute values of the two was calculated, which is the fitting value of the light energy-utilization index. The PAR corresponding to $P_{\text {Nmin }}$ was found to be the measured value of $I_{\mathrm{c}}$, and the PAR corresponding to $P_{\mathrm{Nmax}}$ was the measured value of $I_{\mathrm{m}}$. The LUE slope $y^{\prime}\left(I_{\mathrm{c}}\right)$ value at $I_{\mathrm{c}}$ and the LUE slope $y^{\prime}\left(I_{\mathrm{c}}\right)$ value at $I_{\mathrm{m}}$ were calculated using Eq. 13, and the sum of the absolute values of the two was calculated as the measured value of LUI. The error of LUI fitting value compared to the measured value was $0 \%$ (Table 2).

\section{Discussion}

Model fitting can be used to analyze and predict various reaction characteristics in the process of photosynthesis (Ye et al. 2013b, Herrmann et al. 2020). According to the fitting equation, relevant physiological parameters were calculated. The daily cumulative value of photosynthetic rate and the saturation light intensity $\left(I_{\mathrm{m}}\right)$ of leaves under OSC were higher than those under TSC, that is, leaves under the OSC had a large number of photosynthetic products and could better adapt to strong light conditions. At the same time, LAR and the maximum photosynthetic rate $\left(P_{\mathrm{Nmax}}\right)$ of the leaves with OSC shape were also larger than that of the leaves with TSC shape. The dark respiration rate $\left(R_{\mathrm{D}}\right)$ of the OSC was smaller than that of the TSC. The LUE $_{\max }$ of 'Red Globe' and 'Flame Seedless' leaves under the OSC shape was higher than that of TSC shape.

The diurnal variation of photosynthesis of the two cultivars presented a bimodal curve under the two tree shape conditions. The photosynthetic rate of 'Red Globe' fell between 12:00-18:00 h, while that of 'Flame Seedless' fell between 10:00-16:00 h. The measurement results of stomatal conductance and water vapor pressure deficit show that they are significantly related to the diurnal variation of net photosynthetic rate. The stomatal conductance of 'Red Globe' grape leaves decreased from 12:00 to $16: 00 \mathrm{~h}$ and increased slightly from 16:00 to 18:00 h. The stomatal conductance of 'Flame Seedless' leaves decreased from 10:00 to $13: 00 \mathrm{~h}$ and slightly increased from 13:00 to 16:00 h. The variation trend of intercellular $\mathrm{CO}_{2}$ concentration was similar to stomatal conductance in two cultivars. Therefore, it is believed that high water vapor pressure deficit and leaf stomatal closure at noon are the main reasons for the decrease of photosynthetic rate, while high water vapor pressure deficit and stomatal closure may be caused by high temperature and dryness at noon.

The response curve of net photosynthetic rate to light intensity under low light intensity is a powerful tool to evaluate the photosynthetic characteristics of plants (Ye and Gao 2007). If fitted photosynthetic effective radiation range is different, the resulting apparent quantum efficiency is different. To avoid man-made factors, this study chose the light-compensation point of quantum efficiency in plants using light energy as an index. For the ability of plant leaves to convert light energy into clean energy, intrinsic quantum efficiency has the biggest potential; the use of light energy by plants increases this value (Ye et al. $2013 b)$ The slope $\left(y^{\prime}\right)$ of LUE changes at different PAR and indicates the rate of change of LUE, and the index of LUE of OSC was higher than that of TSC.

The photosynthetic utilization range LAR of leaves 
of the two varieties with OSC was larger than that of the TSC shape, and the saturation light intensity I was higher than that of the TSC shape, which reflected the advantage of OSC in the utilization of strong light in the Junggar basin. When PAR $\geq 28 \mu$ mol(photon) $\mathrm{m}^{-2} \mathrm{~s}^{-1}$, the leaves of 'Red Globe' and 'Flame Seedless' vines were able to start photosynthesis. PAR reached the highest photosynthetic efficiency in leaves when it was about 270-400 $\mu \mathrm{mol}$ (photon) $\mathrm{m}^{-2} \mathrm{~s}^{-1}$, reflecting the higher photosynthetic efficiency of plants in the state of 'light hunger' (Haritha et al. 2017).

Through the fitting calculation of three data models, consistent conclusions were obtained in LAR, potential $P_{\mathrm{Nmax}}, \mathrm{PAR}_{\mathrm{me}}$, etc., and the above results all reflect the superiority of OSC in leaf photosynthesis. The difference of photosynthetic ability between the two varieties was caused by the difference of physiological and biochemical systems between the two varieties, while the difference of photosynthetic ability between the two trees might be caused by the following reasons: (1) The difference of leaf canopy caused differences in microclimate, which resulted in the difference of stomatal opening degree, leaf temperature, and intercellular $\mathrm{CO}_{2}$ concentration. (2) Differences in thinning out secondary branches: TSC requires pruning of the secondary branches, while OSC does not until the leaf area index is above 3 . Some studies have shown that thinning out secondary shoots reduces the stomatal conductance of the first branch leaves, thus decreasing $P_{\mathrm{N}}$ (Pan et al. 2017). (3) The degree of twist between branches that are pulled up and down from trellis is different: OSC has 2-dimensional bending of $30^{\circ}$, and TSC has three-dimensional bending of $90^{\circ}$ in the process of pulling branches up and down from the trellis. In production, TSC often causes sprains of branches, which affects the supply of water and mineral elements to leaves and have adverse effects on leaf photosynthesis. In the early stage, our team evaluated OSC and TSC in the aspects of high light efficiency, labor saving, and coordinated agronomy and machinery (Zhang et al. 2015). This study is a supplement to the research on grape trellis evaluation.

Conclusion: The Gauss, Yezip, and FreundlichEXT model can accurately determine grape leaf photosynthetic parameters and environmental factors affecting these parameters and the possible physiological ecology for grapes and other fruit crops. This type of physiological ecology research can be used to provide reasonable and effective parameters. In this study, OSC was shown to better adapt to the Junggar basin in Xinjiang, China climate environment compared to TSC.

\section{References}

Bassman J.H., Zwier J.C.: Gas exchange characteristics of Populus trichocarpa, Populus deltoides and Populus trichocarpa $\times$ P. deltoides clones. - Tree Physiol. 8: 145-159, 1991.

Bernacchi C.J., Pimentel C., Long S.P.: In vivo temperature response functions of parameters required to model RuBP- limited photosynthesis. - Plant Cell Environ. 26: 1419-1430, 2003.

Bordenave C.D., Rocco R., Maiale S.J. et al.: Chlorophyll $a$ fluorescence analysis reveals divergent photosystem II responses to saline, alkaline and saline-alkaline stresses in the two Lotus japonicus model ecotypes MG20 and Gifu-129. Acta Physiol. Plant. 41: 167, 2019.

Broadley M.R., Escobar-Gutiérrez A.J., Burns A., Burns I.G.: Nitrogen-limited growth of lettuce is associated with lower stomatal conductance. - New Phytol. 152: 97-106, 2001.

Dias-Filho M.B.: Photosynthetic light response of the $\mathrm{C}_{4}$ grasses Brachiaria brizantha and B. humidicola under shade. - Sci. Agric. 59: 65-68, 2002.

Ethier G.J., Livingston N.J.: On the need to incorporate sensitivity to $\mathrm{CO}_{2}$ transfer conductance into the Farquhar-von Caemmerer-Berry leaf photosynthesis model. - Plant Cell Environ. 27: 137-153, 2004.

Galle A., Florez-Sarasa I., El Aououad H., Flexas J.: The Mediterranean evergreen Quercus ilex and the semi-deciduous Cistus albidus differ in their leaf gas exchange regulation and acclimation to repeated drought and re-watering cycles. J. Exp. Bot. 62: 5207-5216, 2011.

Han Y., Hao T.Y., Li Z.Y., Li Y.: Inversion of the fluorescence spectral information of vegetation chlorophyll based on the inverted Gaussian model. - J. Quant. Spectrosc. Ra. 242: 106761, 2020.

Han Z.G., Lei L.M., Han B.P.: [Changes in rapid light curves of Phaeodactylum tricornutum and Prorocentrum dentatum during light-dark cycles.] - J. Trop. Oceanogr. 24: 13-21, 2005. [In Chinese] doi: 10.3969/j.issn.1009-5470.2005.06.003.

Haritha G., Vishnukiran T., Yugandhar P. et al.: Introgressions from Oryza rufipogon increase photosynthetic efficiency of KMR3 rice lines. - Rice Sci. 24: 85-96, 2017.

Harley P.C., Thomas R.B., Reynolds J.F., Strain B.R.: Modelling photosynthesis of cotton grown in elevated $\mathrm{CO}_{2}$. - Plant Cell Environ. 15: 271-282, 1992.

Herrmann H.A., Schwartz J.-M., Johnson G.N.: From empirical to theoretical models of light response curves - linking photosynthetic and metabolic acclimation. - Photosynth. Res. 145: 5-14, 2020.

Li T., Zhang J.F., Chen H.J. et al.: [The ripe grape leaf net photosynthetic rate change under the different water treatment.] - J. Arid Land Resour. Environ. 9: 179-184, 2010. [In Chinese] doi: 10.13448/j.cnki.jalre.2010.09.024.

Li Z.Y., Dong J.H.: [Dynamics model for hydration of cement curing under isothermal condition based on Extended Freundlich.] - Sichuan Build. Sci. 39: 186-189, 2013. [In Chinese] doi: 10.3969/j.issn.1008-1933.2013.01.047.

Majeed Y., Karkee M., Zhang Q. et al.: Determining grapevine cordon shape for automated green shoot thinning using semantic segmentation-based deep learning networks. Comput. Electron. Agr. 171: 105308, 2020.

Meacham-Hensold K., Montes C.M., Wu J. et al.: Highthroughput field phenotyping using hyperspectral reflectance and partial least squares regression (PLSR) reveals genetic modifications to photosynthetic capacity. - Remote Sens. Environ. 231: 111176, 2019.

Murchie E.H., Niyogi K.K.: Manipulation of photoprotection to improve plant photosynthesis. - Plant Physiol. 155: 86-92, 2011.

Na C.: Size-controlled capacity and isocapacity concentration in freundlich adsorption. - ACS Omega 5, 13130-13135, 2020.

Norton A.J., Rayner P.J., Koffi E.N. et al.: Estimating global gross primary productivity using chlorophyll fluorescence and a data assimilation system with the BETHY-SCOPE model. - Biogeosciences 15: 3069-3093, 2019. 
Pan M.Q., Zhang F.X., Zhong H.X. et al.: [Evaluation of high photosynthetic efficiency and facilitation in grape 'single cordon along the ditch obliquely' in Northern China.] J. Fruit Sci. 9: 1134-1143, 2017. [In Chinese] doi:10.13925/j. cnki.gsxb.20160156.

Prado C.D.A., De Moraes J.: Photosynthetic capacity and specific leaf mass in twenty woody species of cerrado vegetation under field conditions. - Photosynthetica 33: 103-112, 1997.

Rascher U., Liebig M., Lüttge U.: Evaluation of instant lightresponse curves of chlorophyll fluorescence parameters obtained with a portable chlorophyll fluorometer on site in the field. - Plant Cell Environ. 23: 1397-1405, 2000.

Reynolds M., Foulkes J., Furbank R. et al.: Achieving yield gains in wheat. - Plant Cell Environ. 35: 1799-1823, 2012.

San Martín F., Kracht W., Vargas T.: Attachment of Acidithiobacillus ferrooxidans to pyrite in fresh and saline water and fitting to Langmuir and Freundlich isotherms. - Biotechnol. Lett. 42: 957-964, 2020.

Tan J., Ding J.L., Zhang J.Y.: [Temporal and spatial variation in temperature in Northern Xinjiang during 1961-2014.] Arid Zone Res. 7: 1-14, 2018. [In Chinese] doi: 10.13866/j. azr.2018.05.23.

van der Tol C., Vilfan N., Dauwe D. et al.: The scattering and re- absorption of red and near-infrared chlorophyll fluorescence in the models Fluspect and SCOPE. - Remote Sens. Environ. 232: 111292, 2019.

Ye Z.P., Gao J.: [Relationship photosynthetic rate of Salvia miltiorrhiza with irradiance at low photon flux densities.] J. Jinggangshan Univ. 28: 47-50, 2007. [In Chinese] doi: 10.3969/j.issn.1674-8085.2007.04.017.

Ye Z.P., Robakowski P., Suggett D.J.: A mechanistic model for the light response of photosynthetic electron transport rate based on light harvesting properties of photosynthetic pigment molecules. - Planta 237: 837-847, 2013 a.

Ye Z.P., Suggett D.J., Robakowski P., Kang H.J.: A mechanistic model for the photosynthesis-light response based on the photosynthetic electron transport of photosystem II in $\mathrm{C}_{3}$ and $\mathrm{C}_{4}$ species. - New Phytol. 199: 110-120, $2013 \mathrm{~b}$.

Zhang F.C., Pan M.Q., Lu C.S. et al: [Diurnal variations and light responses of four grape varieties in Turpan.] - Xinjiang Agric. Sci. 6: 1001-1005, 2011. [In Chinese] doi: 10.6048/j. issn.1001-4330.2011.06.004.

Zhang F.C., Pan M.Q., Wu X.Y. et al.: [Preliminary research on 'single cordon along the ditch obliquely' of grape in cold areas.] - Agr. Res. Arid Area. 5: 68-74, 2015. [In Chinese] doi: 10.7606/j.issn.1000-7601.2015.05.13. 
F.C. ZHANG et al.

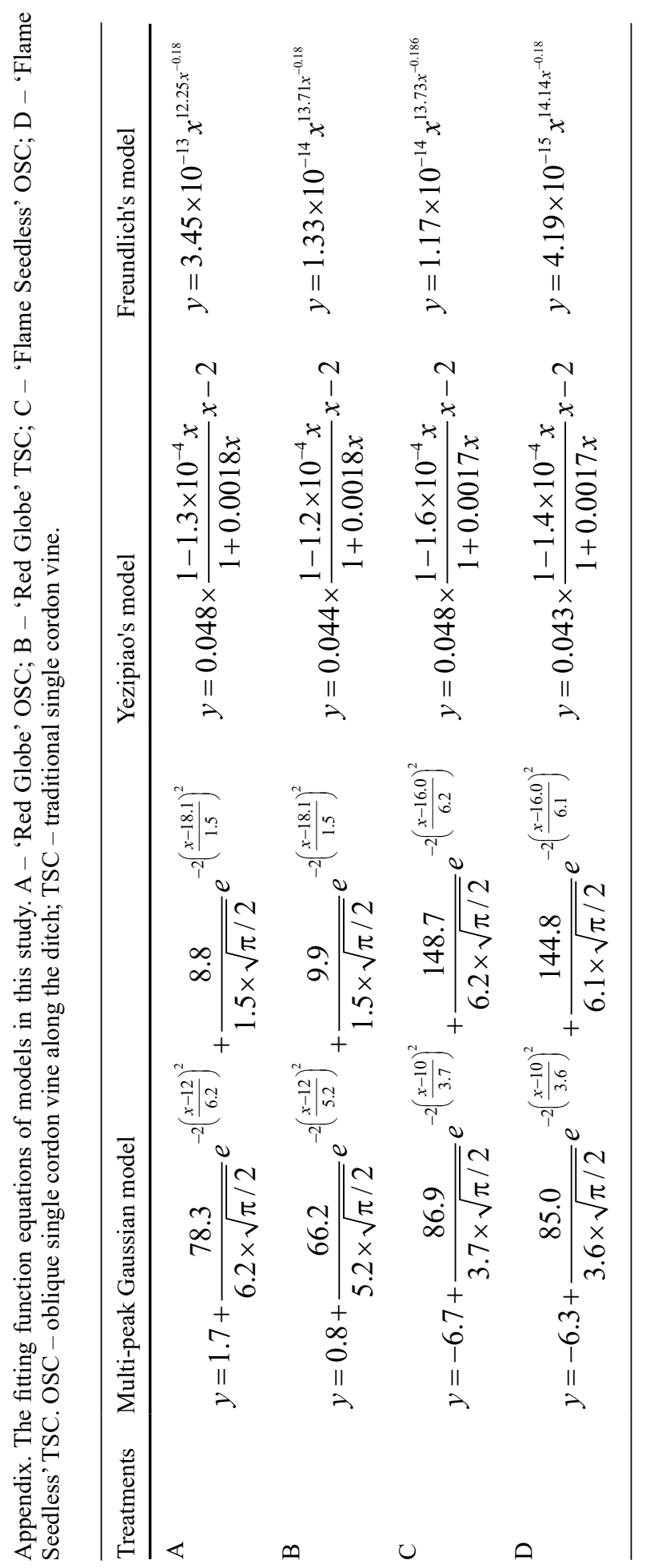

(C) The authors. This is an open access article distributed under the terms of the Creative Commons BY-NC-ND Licence. 